Ann. Biol. anim. Bioch. Biophys., I967, 7 (2), I3I-I43.

\title{
EVOLUTION, AU DEBUT DE LA LACTATION, DE LA SECRÉTION DES PRINCIPAUX ACIDES GRAS DU LAIT ET DE LA CONCENTRATION EN ACIDES GRAS LIBRES DU SANG CHEZ LA VACHE
}

\author{
C. DECAEN et M. JOURNET \\ avec la collaboration technique de Renée LEFAIVRE, Louise Toullec, \\ Y. Manis, A. Hoden et B. Marquis \\ Station de Recherches sur l'Élevage des Ruminants, \\ Centre de Recherches zootechniques et vétérinaires sur les Ruminants, \\ 63 Theix, près Clermont-Ferrand \\ Institut national de la Recherche agronomique
}

\section{SOMMATRE}

La composition en acides gras des matières grasses des laits individuels de 9 vaches correctement alimentées a évolué de façon importante au cours des six premières semaines de lactation. $\mathrm{La}$ proportion d'acides gras longs $\left(\mathrm{C}_{18}: \circ\right.$ et $\left.\mathrm{C}_{18}: \mathrm{I}\right)$ a évolué parallèlement à la teneur en acides gras libres du plasma. Ces deux valeurs ont diminué pour les 7 vaches qui ont vêlé à terme mais elles ont augmenté pour les 2 vaches qui ont vêlé prématurément. La proportion d'acides gras courts $\left(\mathrm{C}_{6} \dot{a}\right.$ $\mathrm{C}_{14}$ ) a évolué en sens inverse.

Ces changements de la composition des matières grasses du lait semblent dus à la mobilisation des réserves lipidiques, c'est ce qui est bien montré par les variations parallèles du poids vif, du bilan énergétique, de la teneur en acides gras longs du lait observées sur un même animal ainsi qu'entre animaux.

La teneur en matières grasses du lait, au cours des six premières semaines de lactation a évolué pour presque toutes les vaches parallèlement à la teneur en acides gras longs du lait exprimée en $\mathrm{g}$ par $\mathrm{kg}$ de lait.

Les acides gras libres du plasma pourraient être des précurseurs importants des matières grasses du lait au début de la lactation. D'une façon plus générale leur étude semble particulièrement intéressante chez la vache laitière, en particulier pour connaître l'orientation du métabolisme lipidique.

\section{INTRODUCTION}

La sécrétion des matières grasses du lait présente une évolution caractéristique chez la vache correctement alimentée : le taux butyreux diminue en moyenne jusqu'à la $6^{\mathbf{e}}$ semaine, tandis que la quantité totale de matières grasses sécrétées aug- 
mente durant les 2 dernières semaines (JOURNET et JARRIGE, I960 ; DECAEN et PouTous, I967). La composition des triglycérides du lait varie elle aussi : la proportion d'acides gras courts augmente tandis que celle des acides gras longs diminue (DECAEN et ADDA, I966). Ces variations pourraient résulter d'une mobilisation des réserves corporelles de la vache.

Or, on sait que 1'hydrolyse des triglycérides du tissu adipeux libère dans le sang des acides gras libres qui sont en majorité des acides gras longs en $\mathrm{C}_{16}$ et $\mathrm{C}_{18}$. Depuis les travaux de Dole (I956) et de Gordon et CHERCKEs (1956) les variations de la concentration en acides gras libres du plasma ont été beaucoup étudiées chez le monogastrique mais assez peu chez les Ruminants et en particulier chez la vache (KRONFELD, I 965). Ils représentent une faible part des acides plasmatiques totaux (GARToN et DunCAN, I964), mais ils ont un "turn-over" rapide (DOLE, I96I).

Nous avons cherché à relier les variations de la composition en acides gras des matières grasses du lait à celles des acides gras libres du plasma au cours des 6 premières semaines après le vêlage.

\section{MATÉRIEI, ET MÉTHODES}

\section{Animaux et régimes alimentaires}

Nous avons utilisé 9 vaches : 6 Frisonnes, 2 Pies Rouges et I Normande ayant vêlé entre le début décembre 1964 et le début mars 1965. Leur production laitière (moyenne journalière au cours des 6 premières semaines) a varié selon les vaches de $\mathrm{I} 6,8$ à $31,6 \mathrm{~kg}$ et la teneur en matières grasses de 34,7 à $46,4 \mathrm{~g}$ p. I 000 . Trois de ces vaches ont reçu une ration de fourrage composée de foin de luzerne normal et de foin de luzerne condensé (broyé et aggloméré dans la proportion de i à 3 environ et les 6 autres une ration de foin de luzerne normal, d'ensilage d'herbe et de $20 \mathrm{~kg}$ de betteraves. Toutes les vaches recevaient avant le vêlage $2 \mathrm{~kg}$ d'aliment concentré, et après le vêlage cette quantité a été accrue au cours du premier mois de lactation pour couvrir les besoins.

Deux de ces vaches ont présenté certaines particularités : l'une, la 0046 , a vêlé to jours avant le terme et a produit une quantité de lait inférieure d'environ $40 \mathrm{p}$. Ioo à celle de la lactation précédente; l'autre, la 0103 , a avorté après un repos mammaire de 2 semaines seulement. Les résultats de ces 2 vaches, au vêlage prématuré, seront considérés séparément et comparés à ceux des 7 autres. Ces dernières ont eu une production laitière qui a évolué normalement et qui a été maximum entre la $4^{\mathrm{e}}$ et la $6^{\mathrm{e}}$ semaine de lactation. Plusieurs accidents cependant sont à noter : une fièvre de lait au vêlage sur la vache 9014 et des indigestions sur la vache 0056 (la $2^{\mathbf{e}}$ semaine après le vêlage), sur la vache 9I6r (la $5^{\mathrm{e}}$ semaine), sur la vache 9014 (la $4^{\mathrm{e}}$ semaine).

\section{Mesures}

Les vaches ont été alimentées individuellement et les quantités offertes et refusées de chaque aliment ont été mesurées chaque jour. La valeur énergétique (UF) des foins a été estimée à partir des valeurs du coefficient d'utilisation digestive obtenues sur des moutons maintenus en cages à bilan ; celle des autres aliments a été estimée à partir de leur teneur en cellulose brute. Le bilan énergétique a été calculé par différence entre les besoins des animaux (entretien + production) et les apports de la ration.

La quantité de lait a été pesée à chacune des 2 traites journalières; le taux butyreux a été mesuré par la méthode Gerber, chaque jour, sur un échantillon de lait représentatif des traites du matin et du soir. La composition en acides gras des triglycérides du lait a été mesurée, chaque semaine, sur un échantillon moyen du lait de 4 traites consécutives, par chromatographie en phase gazeuse d'esters méthyliques et butyliques (cf. DECAEN et ADDA, 1966). A partir de la quantité de lait sécrétée, de la teneur de ce lait en matières grasses et de la composition en acides gras de ces matières grasses, nous avons estimé les quantités journalières des différents acides gras majeurs sécrétés par jour (moyenne des 2 jours). 
La concentration des acides gras non estérifiés dans le plasma (Dole et MEINERTz, I960) a été déterminée sur des échantillons de $100 \mathrm{ml}$ de sang prélevés au niveau de la veine jugulaire une fois par semaine, le même jour ( $\mathrm{I}^{\mathrm{er}}$ jour) que le lait destiné à la détermination de la composition des triglycérides. Trois prélèvements journaliers de sang ont été effectués à $6 \mathrm{~h}$, avant le premier repas, à Io heures, soit 3 heures après le premier repas de foin de luzerne condensé ou d'ensilage et à ז6 heures. Les animaux ont été pesés une fois par semaine, à jour et heure fixes.

\section{RÉSULTATS}

La sécrétion des matières grasses des 7 vaches normales a évolué en moyenne de la façon suivante (fig. I) : la teneur en matières grasses a diminué de II g p. I 000 au cours des 6 premières semaines de lactation; la production de matières grasses a augmenté légèrement de $3,5 \mathrm{p}$. Ioo et a été maximum à la $4^{\mathbf{e}}$ semaine de lactation ; la composition des matières grasses a évolué selon le schéma que nous avons décrit
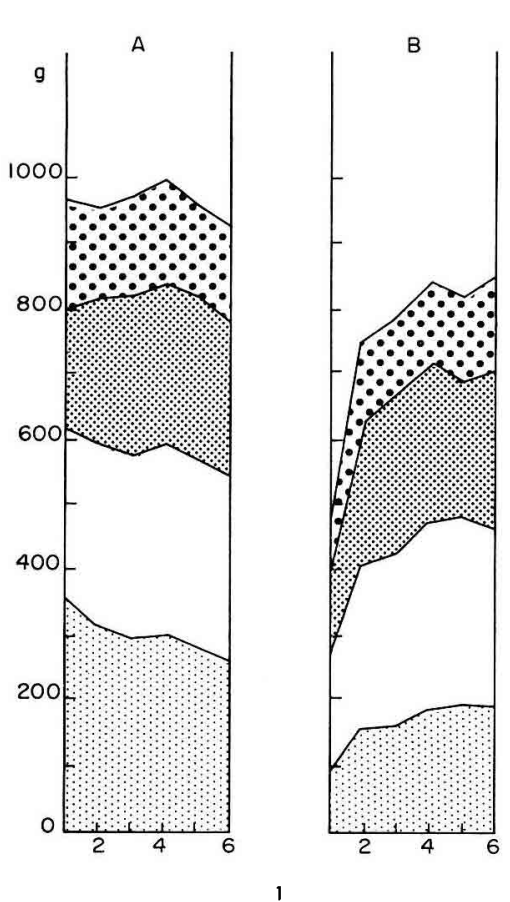

Quantités d'acides gras $(\mathrm{g})$

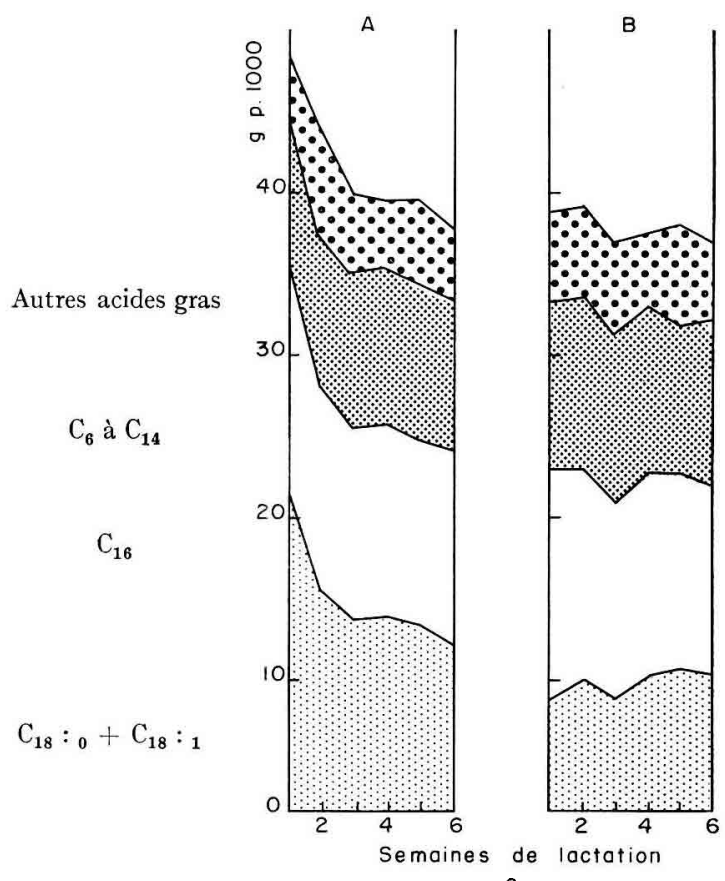

2

Teneurs en acides gras ( $\mathrm{g}$ par $\mathrm{kg}$ de lait)

FIG. I. - Évolution des acides gras des triglycérides du lait (sous forme d'esters méthyliques) de 7 vaches $(A)$ au vêlage normal et de 2 vaches $(B)$ au vêlage prématuré

précédemment (DECAEN et ADDA, I966). Elle n'a pas été différente pour les deux régimes alimentaires (tabl. I). La proportion d'acides gras longs (stéarique et oléîque) décroît, passant de 36,4 à 27,2 p. Ioo en 6 semaines; inversement la proportion d'acides gras courts $\left(C_{6}\right.$ à $\left.C_{14}\right)$ augmente, passant de $I 7, I$ à 25,4 $p$. Ioo en 5 semaines. La proportion d'acide palmitique varie peu : elle est de 29 p. Ioo en moyenne. L'ac- 


\begin{tabular}{|c|c|c|c|c|}
\hline & & & 0 & 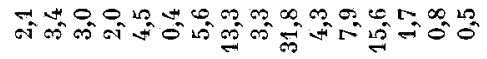 \\
\hline & & 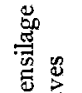 & 15 & 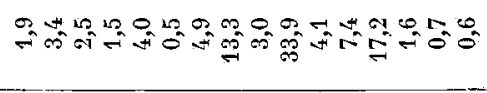 \\
\hline$\frac{\sqrt[3]{3}}{3}$ & 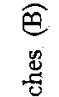 & 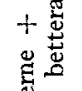 & 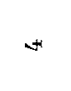 & 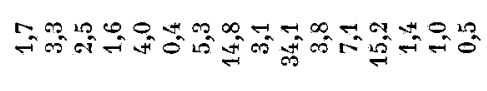 \\
\hline 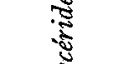 & $\stackrel{4}{i}$ & $\begin{array}{l}N+ \\
\Xi\end{array}$ & $\infty$ & 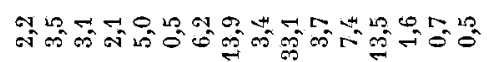 \\
\hline$\frac{8}{3}$ & & 政 & ov & 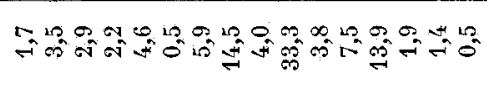 \\
\hline & & & $r$ & 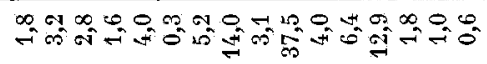 \\
\hline $\begin{array}{c}8 \\
8 \\
8 \\
8\end{array}$ & & & 0 & 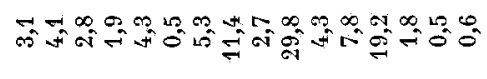 \\
\hline . & & 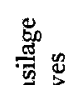 & is & से \\
\hline & $\widehat{\bigsqcup}$ & 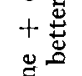 & 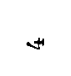 & 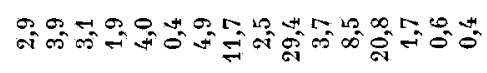 \\
\hline & 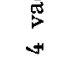 & 尝 & $\infty$ & 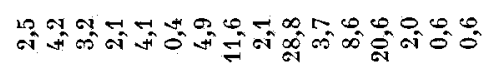 \\
\hline 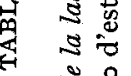 & & 证 & $\infty$ & $\Rightarrow$ \\
\hline . हू & & & + & 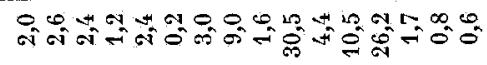 \\
\hline & & & 0 & 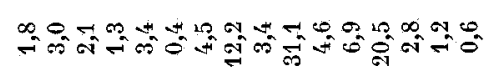 \\
\hline हूँ & & 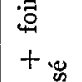 & 10 & 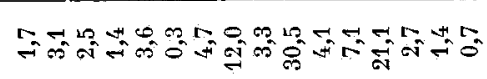 \\
\hline 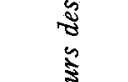 & 这 & हี छั & + & 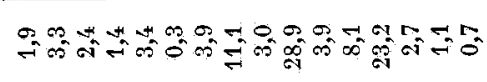 \\
\hline 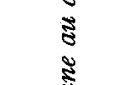 & $\frac{\pi}{\infty}$ & 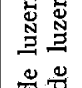 & $\infty$ & 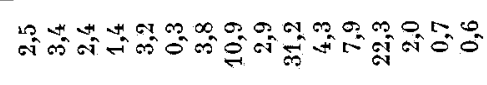 \\
\hline हैँ & & 沾 & G & 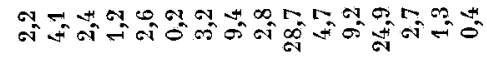 \\
\hline 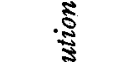 & & & $r$ & 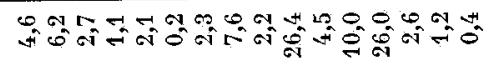 \\
\hline & & 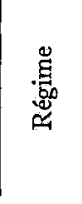 & 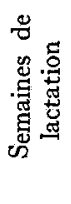 & 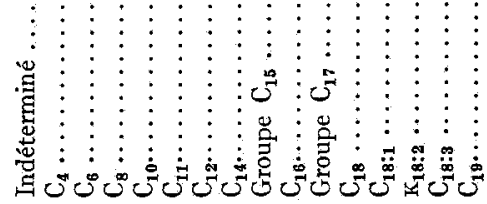 \\
\hline
\end{tabular}


croissement de la quantité de matières grasses sécrétées est due aux acides gras courts dont la production croît de $46 \mathrm{p}$. Ioo (de I69 à $249 \mathrm{~g}$ ), celle d'acides gras longs diminuant d'environ $30 \mathrm{p}$. Ioo (de 357 à $256 \mathrm{~g}$ ). Quant à la diminution de la teneur en matières grasses du lait elle est due à celle de la teneur en acides gras longs essentiellement ainsi qu'à celle en $\mathrm{C}_{16}$, la teneur en acides gras courts restant constante au cours des 6 semaines de la lactation.

La sécrétion des matières grasses des 2 autres vaches, au comportement aberrant, a évolué de façon très différente (fig. I). La quantité de matières grasses sécrétées est faible la première semaine et elle augmente ensuite entre la $\mathrm{I}^{\mathrm{re}}$ et $\mathrm{la} 4^{\mathrm{e}} \mathrm{se}-$ maine de lactation. Au contraire, la teneur en matières grasses évolue très peu : elle baisse seulement de 2 points entre la $\mathrm{I}^{\mathrm{re}}$ et $\mathrm{la} 6^{\mathrm{e}}$ semaine. La composition des matières grasses évolue en sens inverse des 7 autres vaches (tabl. I) : la proportion d'acides gras longs augmente au lieu de diminuer, celle d'acides gras courts varie peu, celle d'acide palmitique diminue de la $\mathrm{I}^{\mathrm{re}}$ à $1 \mathrm{a} 2^{\mathbf{e}}$ semaine et reste constante ensuite jusqu'à la $6^{\theta}$ semaine. De ce fait, $1^{\prime}$ accroissement de la quantité de matières grasses sécrétées entre la $\mathrm{I}^{\mathrm{re}}$ et la $6^{\mathrm{e}}$ semaine est due en partie aux acides gras courts dont 1a production croît de $80 \mathrm{p}$. Ioo (I34 à $243 \mathrm{~g}$ ), à l'acide palmitique (I 80 à $275 \mathrm{~g}$ ) et surtout aux acides gras longs dont la production fait plus que doubler (93 à I99 g). $L_{a}$ faible évolution de la teneur en matières grasses vient de ce que la teneur en acides gras par kg de lait de chacun des groupes précédents n'a pas évolué de façon importante.

\section{Concentration en acides gras libres $d u$ sang}

La concentration en acides gras libres présente une évolution considérable au cours des 6 premières semaines de la lactation, mais elle est très différente pour les 7 vaches normales et pour les 2 autres vaches. Pour les premières, elle augmente brutalement au moment du vêlage (les valeurs observées le matin sont de trois à cinq fois plus élevées après qu'avant la mise bas), puis elle diminue rapidement de

TABLEAU 2

Teneur en acides gras libres du sang (microéquivalents par litre de plasma)

Prélèvements effectués à 6 heures le matin

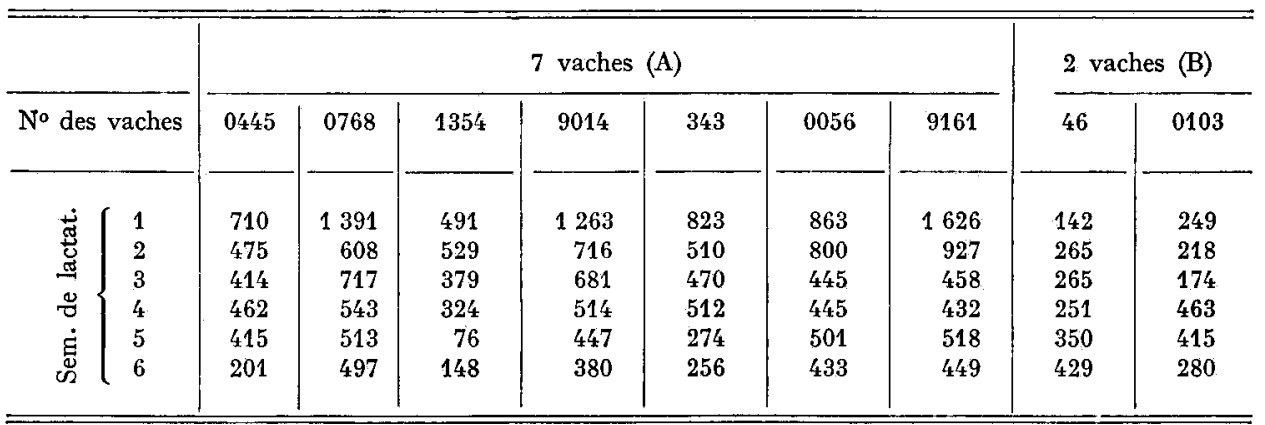

la $\mathrm{I}^{\mathrm{re}} \mathrm{a}$ la $3^{\mathrm{e}}$ semaine de lactation et plus lentement pour atteindre à la $6^{\mathrm{e}}$ semaine des valeurs à peine plus élevées qu'avant le vêlage. Au contraire pour les 2 autres vaches, elle ne varie pas au vêlage et elle augmente au cours des 6 premières semaines 
de la lactation. Pour les prélèvements du matin la concentration en acides gras libres est plus élevée surtout avec les 7 vaches normales et elle varie plus, d'une semaine de lactation à l'autre, que pour les deux autres prélèvements. Cette différence entre heures de prélèvements est beaucoup moins importante pour les 2 autres vaches. (fig. 2).

\section{Bilan énergétique et poids vif}

Pour les 7 vaches normales le bilan énergétique est très nettement négatif dans les jours qui suivent le vêlage, mais il s'améliore ensuite et devient équilibré après 5 ou 6 semaines de lactation; cela est bien montré également par l'évolution du poids vif. En revanche, pour les 2 autres vaches, le bilan énergétique nettement positif juste après le vêlage diminue pour s'équilibrer à la $\sigma^{e}$ semaine; le poids vif évolue très peu. Ainsi, pour tous les animaux le bilan énergétique évolue parallèlement à la teneur en acides gras longs du lait et à la teneur en acides gras libres du plasma, ce que montre bien la figure 3 sur laquelle figurent les valeurs individuelles. L'évolution du poids vif bien qu'elle se fasse en moyenne dans le même sens est beaucoup moins parallèle (fig. 2).
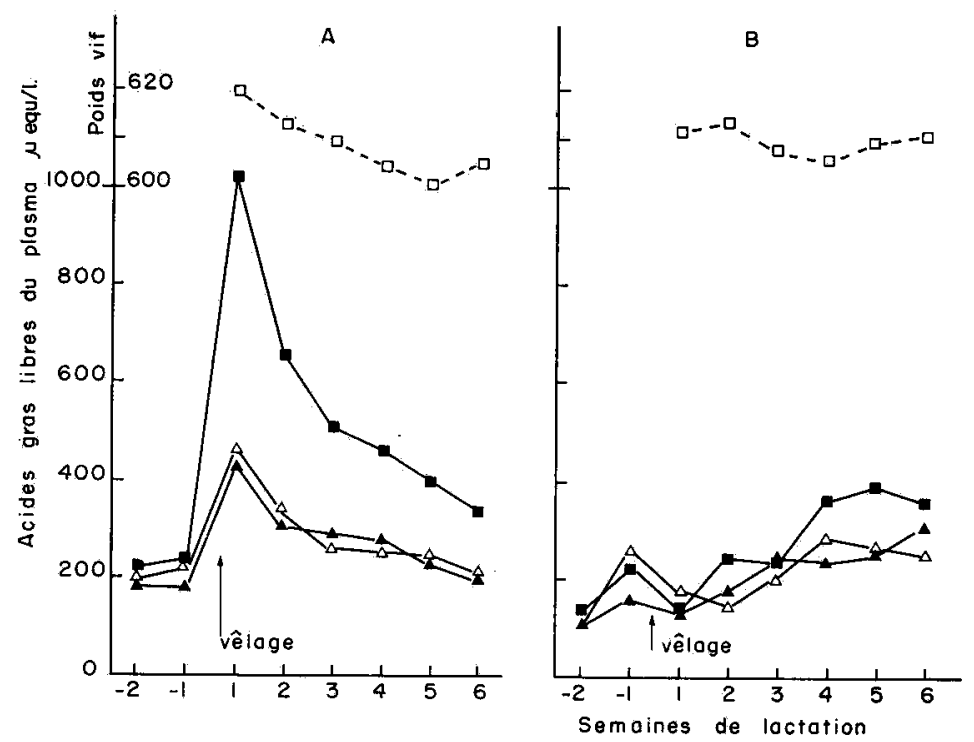

Fig. 2. - Évolution du poids vif des animaux $\square$ et de la teneur en acides gras libres du plasma de 7 vaches $(A)$ au vêlage normal et de 2 vaches $(B)$ au vêlage prématuré

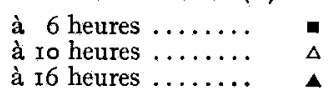

\section{Liaisons observées}

L'évolution au cours des 6 premières semaines de la lactation a permis de mettre en évidence des liaisons positives entre les 3 variables précédentes, teneuren acides gras longs du lait, teneur en acides gras libres du plasma, bilan énergétique, ainsi 
qu'avec le taux butyreux. Nous avons mesuré l'intensité de ces liaisons intra-individuelles en calculant pour chaque animal le coefficient de corrélation entre ces variables prises 2 à 2 , sur les 6 valeurs correspondant aux 6 premières semaines de lactation.
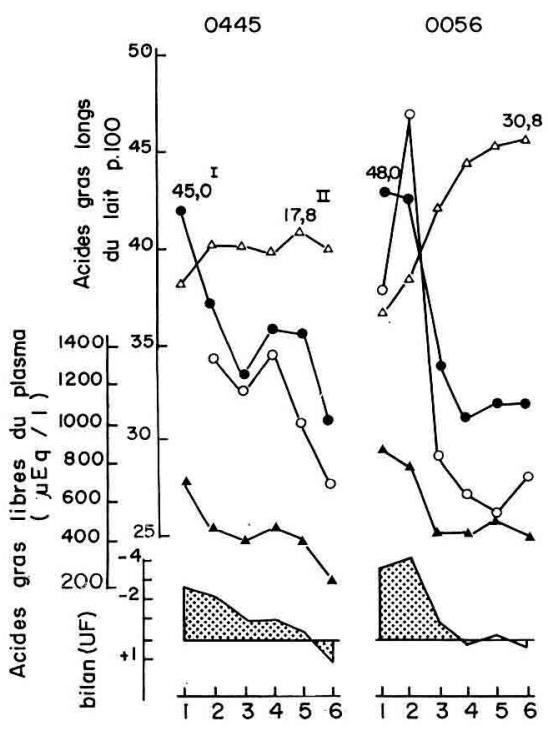

0768

1354

9161

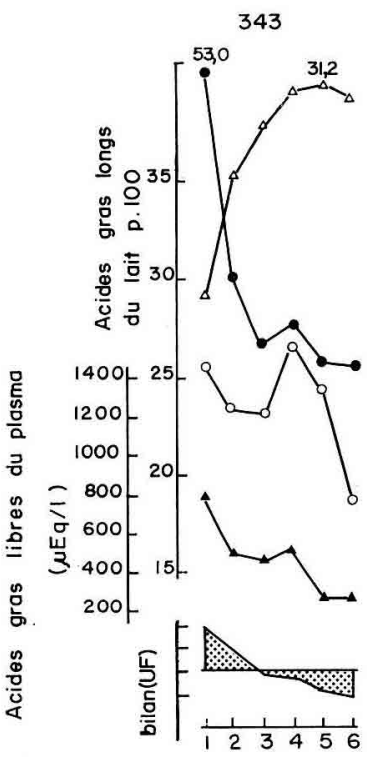

\section{Semaines}
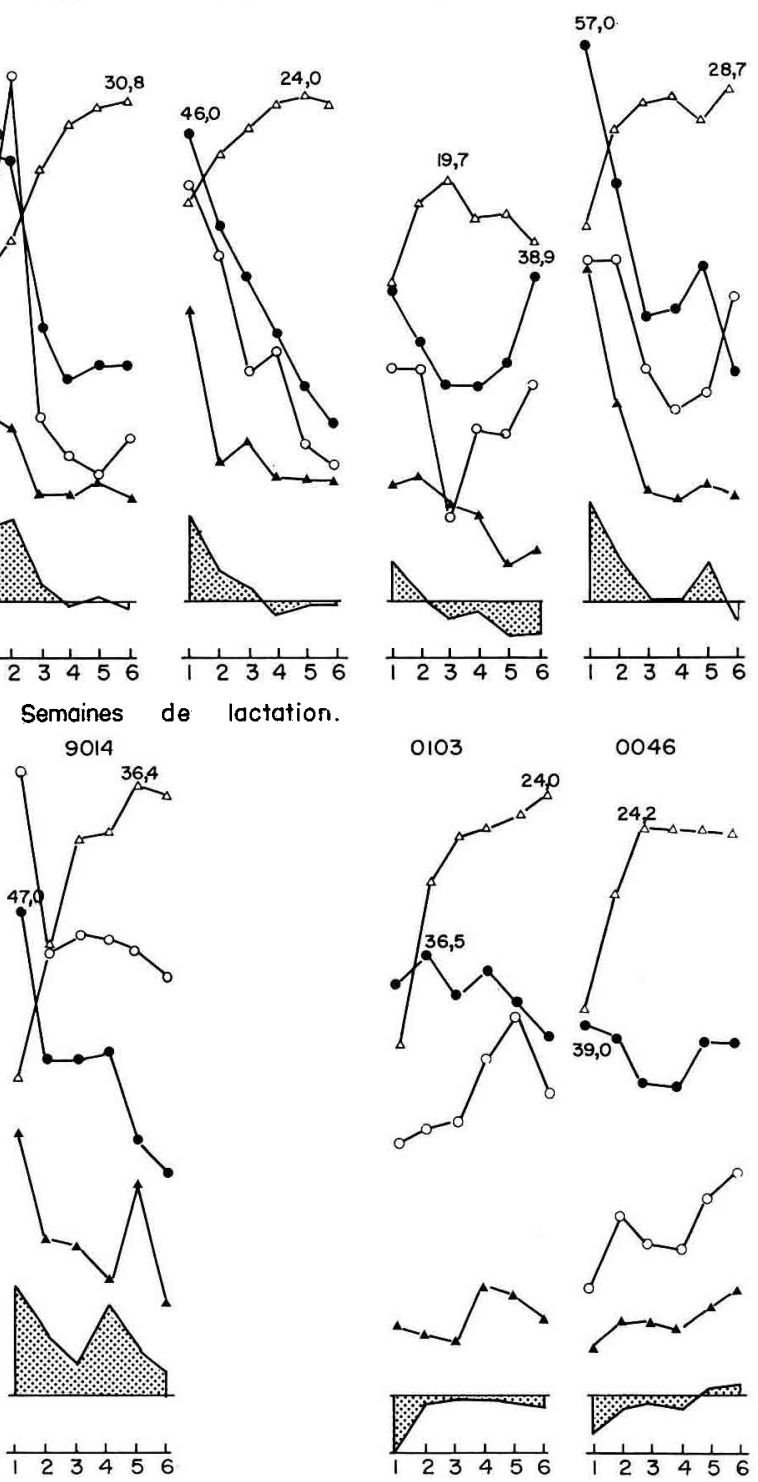

0046

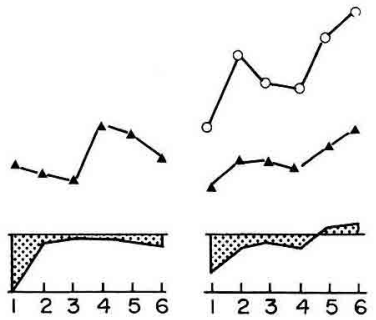

FIG. 3. - Evolution comparée pour chaque vache de la quantité de lait produite $\Delta ;$ du taux butyreux $\bullet ; d e$ proportion d'acides gras longs du lait $\mathrm{O}$; de la teneur en acides gras libres du plasma à 6 heures $\Delta ;$;et du bilan énergétique (bilanUF).
Échelle
$\begin{aligned} \text { Échelle de la quantité de lait ( } \mathrm{kg} \text { ) } \ldots \ldots \ldots & =2 \mathrm{~mm} \\ - \text { du taux butyreux (I } \mathrm{g} \text { p. I 0o0) } \ldots \ldots \ldots . & =2 \mathrm{~mm}\end{aligned}$

(I) Valeur maximum du taux butyreux (g p. I ooo).

(II) - de la quantité de lait (kg). 
Nous avons également regardé si ces liaisons positives existaient également entre animaux soit pour une semaine déterminée de la lactation, première ou deuxième, soit pour la moyenne des 6 semaines. Le tableau 3 donne les coefficients de corrélation.

\section{TABLEAU 3}

Liaisons entre le taux butyreux, la proportion d'acides gras longs du lait, la teneur en acides gras libres du sang, le bilan énergétique

Coefficients de corrélation

\begin{tabular}{|c|c|c|c|}
\hline \multicolumn{3}{|c|}{$\begin{array}{l}\text { Inter-individuels } \\
n=9 \text { vaches }\end{array}$} & $\begin{array}{c}\text { Intra-individuels } \\
n=6 \text { semaines } \\
\text { de lactation }\end{array}$ \\
\hline $\begin{array}{l}\text { Acides gras longs du lait } \\
\text { et acides gras libres du sang }\end{array}$ & $\begin{array}{c}1^{\mathrm{re}} \text { semaine } \\
\text { de lactation } \\
2^{\mathrm{e}} \text { semaine } \\
6 \text { premières semaines }\end{array}$ & $\begin{array}{l}0,85 \\
0,78 \\
0,73\end{array}$ & 0,50 à 0,90 (9 vaches) \\
\hline $\begin{array}{l}\text { Acides gras libres du sang } \\
\text { et bilan énergétique (UF) }\end{array}$ & $\begin{array}{c}1^{\mathrm{re}} \text { semaine } \\
\text { de lactation } \\
2^{\mathrm{e}} \text { semaine } \\
6 \text { premières semaines }\end{array}$ & $\begin{array}{l}0,90 \\
0,69 \\
0,81\end{array}$ & $\begin{array}{c}0,83 \text { à } 0,98 \text { ( } 8 \text { vaches) } \\
0,29 \text { ( } 1 \text { vache) }\end{array}$ \\
\hline $\begin{array}{l}\text { Taux butyreux } \\
\text { et proportion d'acides gras longs }\end{array}$ & 6 premières semaines & 0,31 & $\begin{array}{l}0,43 \text { à } 0,98 \text { ( } 7 \text { vaches) } \\
0,27 \text { et } 0,09 \text { ( } 2 \text { vaches }\end{array}$ \\
\hline
\end{tabular}

Les liaisons intra-individuelles sont élevées pour tous les animaux entre la proportion d'acides gras longs du lait et la teneur en acides gras libres du sang d'une part, et la teneur en acides gras libres du sang et le bilan énergétique d'autre part, à l'exception d'un animal pour cette dernière liaison. La liaison entre teneur en matières grasses et proportion d'acides gras longs du lait est positive mais variable en intensité selon les animaux; elle est non significative pour les 2 vaches au vêlage prématuré.

Les liaisons inter-individuelles demeurent très élevées pour les 2 premières liaisons ci-dessus ; par contre, pour la teneur en matières grasses et la proportion d'acides gras longs, elle est très faible et non significative.

\section{DISCUSSION}

D'après les résultats de cette expérience nous pouvons avoir la conception suivante sur la sécrétion des matières grasses du lait au début de la lactation : la mobilisation des réserves lipidiques entraînée par un bilan énergétique négatif et la libération des acides gras libres dans le sang qui en résulte, peuvent expliquer l'évolution de la composition en acides gras des matières grasses du lait et, en partie, l'évolution du taux butyreux; ce qu'illustre la figure 4 . En effet, la mobilisation des lipides cor- 
porels est mise en évidence par la variation du poids vif et de façon beaucoup plus précise par celle de la teneur en acides gras libres du sang dont la teneur a évolué de façon très parallèle au bilan énergétique. Lorsque le bilan énergétique a été négatif et, en conséquence, la mobilisation des lipides corporels importante, le lait a eu une teneur élevée en acides gras longs et inversement. Cette liaison a été observée au cours des 6 premières semaines de la lactation et dans l'ensemble pour toutes les vaches, que le bilan énergétique augmente pour les vaches normales ou qu'il diminue pour les autres. Cette liaison se retrouve également lorsqu'on compare les vaches entre elles : celles qui ont eu un déficit énergétique élevé et une teneur en acides gras libres élevée, ont sécrété un lait plus riche en acides gras longs. Cette origine des acides gras longs à partir des lipides corporels au début de la lactation a également été montré chez la truie par SALMON-LEGAGNEEUR I965. La preuve cependant ne pourrait en être donnée que par un marquage des acides gras libres et en suivant la variation des dépôts adipeux.
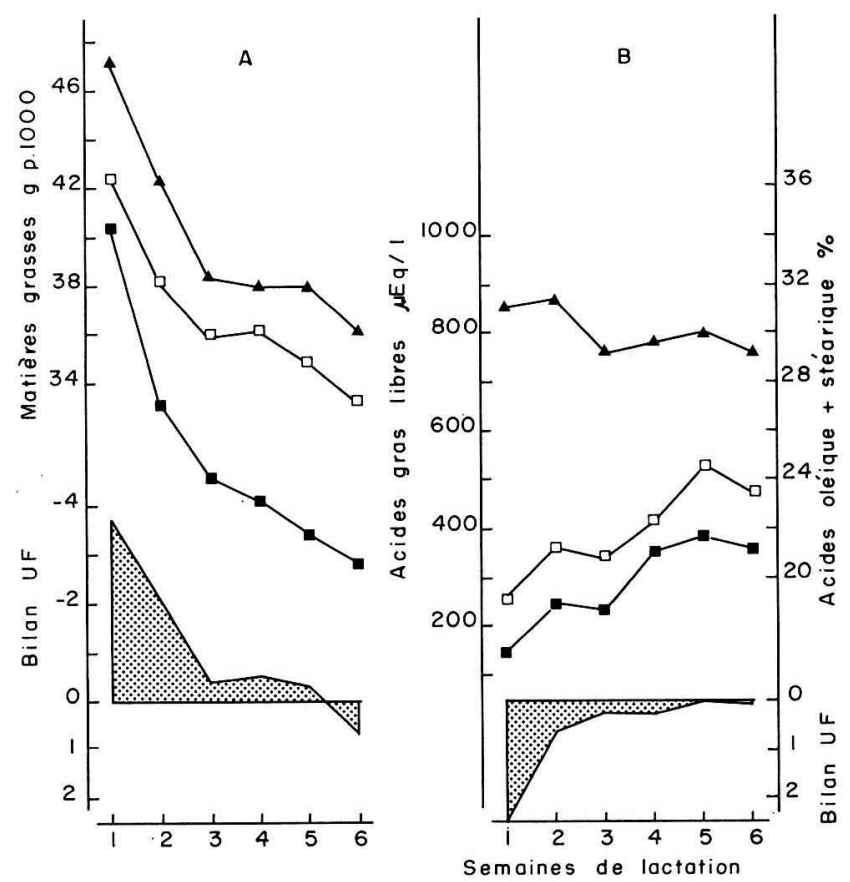

FIG. 4. - Évolution comparée du taux butyreux ^ ; de la proportion d'acides gras longs du lait $\square$; de la teneur en acides gras libres du plasma d 6 haures $\mathbf{0}$; el du bilan énergétique (bilan UF).

$$
\begin{aligned}
& A: 7 \text { vaches au vêlage normal } \\
& B: 2 \text { vaches au vêlage prématuré }
\end{aligned}
$$

L'évolution du taux butyreux, au cours des 6 premières semaines de lactation, a été due, presque uniquement, à celle de la teneur en acides gras longs que ce soit pour les vaches normales dont le taux butyreux a diminué en moyenne de ro points, ou pour les autres dont le taux butyreux a peu varié. En effet, la teneur en acides gras courts n'a pas évolué et celle en acide palmitique, assez peu et dans le sens de celle en acides gras longs. En conséquence, d'après l'hypothèse émise ci-dessus sur l'origine des acides gras longs du lait, on peut donc penser que les taux butyreux, 
généralement élevés au vêlage et décroissants ensuite, sont dus à une mobilisation đes réserves corporelles consécutive à une sous-alimentation énergétique importante au vêlage et qui diminue ensuite.

L'origine des acides gras longs du lait à partir des acides gras libres du plasma ne peut être prouvée par cette étude qui est simplement descriptive. Cependant, les fortes liaisons positives intra-individuelles et inter-individuelles observées la rendent probable, tout au moins pendant cette période du début de la lactation. Le rôle des acides gras libres dans la synthèse de matières grasses du lait est considéré comme négligeable par la plupart des auteurs (GLAscock, I958; BARRY et al., I963; LASCELLES et al., I964), bien que LAURYSSENS et al. Ig6I) aient montré que la mamelle incorpore rapidement dans les glycérides du lait le stéarate injecté dans le sang sous forme libre ou en combinaison avec l'albumine; et que HARTMANN et LASCELIES (I964) et KRONFELD (I965) aient observé des différences artério-veineuses d'acides gras libres pour des teneurs supérieures à $300 \mu$ éq./litre. La plupart des auteurs ont montré que ce sont surtout les acides gras estérifiés du sang, sous forme de chylomicrons et de lipoprotéines, qui sont les principaux précurseurs des acides gras longs du lait. (Glascock, I958; BARRY et al., I963; LASCELLES, I964; STORRY et Rook, I964).

Comme nous n'avons pas mesuré la concentration des différentes fractions lipidiques du sang il est possible que celle-ci ait évolué dans le même sens que celle en acides gras libres; DunCAN et GARTON (I963) ont d'ailleurs observé une lipémie plus élevée après le vêlage qu'avant. Cependant, HARTMANN et LASCELLEs (I965) ont observé que le jour suivant le vêlage, le taux des lipides sanguins était relativement faible alors que celui des acides gras libres était très élevé. On peut donc penser que ce sont bien les acides gras libres sanguins qui sont, au début de la lactation, les précurseurs des acides gras longs des matières grasses du lait.

La libération des acides gras libres dans le sang n'est peut-être pas due uniquement à un déficit énergétique (DECAEN et ADDA, résultats non publiés). On sait que certaines hormones, l'adrénaline, le glucagon, l'hormone somatotrope, l'ocytocine augmentent la concentration des acides gras libres dans le sang alors que 1'insuline l'abaisse (WINKLER et al., I964, KRONFELD, I965, KNOBIL, I964, BALASSE et RASIO, I965). Cependant certaines hormones agissant sur la sécrétion du lait, telle que la prolactine d'après STEINBERG et VAUGHAN, n'auraient pas d'action. En revanche, d'après WILIIANS et al. (I965) la prolactine purifiée provoquerait une libération d'acides gras libres dans le sang. Celle-ci a été également observée par REISs (I947) chez la ratte et chez la femme. Il est possible également que la libération des acides gras libres dans la sang provienne d'une disponibilité en glucose insuffisante (MCCLYMONT et VALLANCE, I 962) : les besoins en glucose de la vache laitière en lactation sont élevés (HEAD et al., I964) et les apports de précurseurs en glucose sont probablement plus faibles au départ de la lactation du fait de l'appétit faible des animaux et de la faible proportion d'aliments concentrés dans la ration.

Les résultats de cette expérience montrent, sans exclure la possibilité d'une intervention hormonale directe, que l'évolution de la sécrétion des matières grasses au début de la lactation peut s'expliquer par les variations du niveau des apports énergétiques de la ration. Au cours des premières semaines la sous-alimentation est fréquente et difficile à éviter; elle résulte d'un brusque accroissement des besoins énergétiques qui sont maximum très tôt après le vêlage (JouRNET et JARRIGE, I960) 
et d'un appétit encore faible (HUTH, I960; HuTTON I963; JOURNET, résultats non publiés). La perte de poids vif qui en résulte serait surtout due aux lipides corporels (FLATt et al., I965). La perte de poids vif de $20 \mathrm{~kg}$ que nous avons observée sur les vaches normales est tout à fait courante et même inférieure à ce qu'on observe normalement sur des troupeaux bien conduits. Dans les conditions de la pratique les modifications de la composition du lait devraient encore être plus importantes. Il serait cependant nécessaire pour avoir la preuve que les variations observées de la teneur en acides gras libres du sang chez les vaches en début de lactation sont dues uniquement à celles du niveau énergétique qu'une expérimentation soit effectuée

\section{Intérêt $d u$ dosage des acides gras libres $d u$ sang chez les Ruminants}

La mesure de la teneur en acides gras libres du sang par la méthode de DOLE (I960) est facile et rapide. Elle peut, de ce fait, être un critère simple de la mobilisation des réserves corporelles de l'animal et en particulier de la vache laitière en début de lactation. Les variations du poids vif en donnent une estimation très imprécise. En effet, au début de la lactation, le poids de contenu digestif croît en même temps que la quantité d'aliment ingérée (JoURNET, résultats non publiés).

La teneur en acides gras libres est susceptible de servir de critère non seulement de l'amaigrissement, mais aussi de l'engraissement des animaux. Les valeurs que nous avons obtenues lorsque les animaux étaient en bilan énergétique équilibré ont été de $35^{\circ}$ à $450 \mu$ éq. 11 ; mais lorsque les vaches ont été en bilan positif nous avons

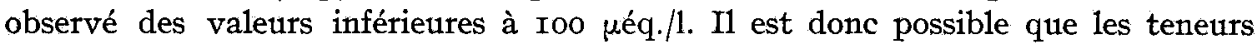
soient d'autant plus faibles que l'animal s'engraisse rapidement. L'estimation de la lipogenèse ou de la dipolyse présente chez le ruminant, et chez la vache tout particulièrement, un intérêt d'ordre plus général. VAN SoEST (I963) a attiré l'attention sur le rôle des facteurs de mobilisation des réserves lipidiques de la vache pour expliquer certaines variations du taux butyreux, en plus du rôle direct joué par certains précurseurs d'origine digestive des matières grasses du lait dont l'acide acétique en particulier. Les relations que nous avons observées entre la teneur en acides gras libres du sang, la proportion d'acides gras longs du lait et le taut butyreux confirment cette hypothèse.

En outre, la détermination de la teneur en acides gras libres du sang doit être un moyen très intéressant pour connaître l'orientation du métabolisme lipidique de la vache laitière, soit vers le dépôt de graisses corporelles, soit vers la sécrétion de matières grasses par la mamelle. Celle-ci doit dépendre de la nature des produits terminaux de la digestion entrant dans le circuit sanguin et notamment de 1'importance relative des pools glucose et acide acétique.

Ręu pour publication en février 1967.

\section{SUMMARY}

CHANGES IN SECRETION OF THE MAIN FATTY ACIDS IN MILK AND THE CONCENTRATION OF FREE FATTY ACIDS IN BLOOD OF COWS AT THE START OF LACTATION

Changes in long-chain fatty acid composition of milk fat and the contents of free fatty acids in plasma were studied during the first 6 weeks of lactation in 9 cows fed correctly. At the same time changes in yields of butterfat and milk and its fat content, and in energy balance and liveweight were followed. 
Seven cows (A) had calved normally, but because of their high milk yield they were underfed during the first weeks of lactation. By contrast, 2 cows (B) which had calved prematurely produced little milk at the start of lactation and were overfed.

I. Fatty acid composition of milk fat changed considerably. For the (A) cows the proportion of long-chain fatty acids $\left(\mathrm{C}_{18}: 0+\mathrm{C}_{18}: \mathrm{I}\right)$ fell, from $3^{6}$ to 27 per cent from the first to the sixth week of lactation. That of short-chain fatty acids $\left(\mathrm{C}_{6}\right.$ to $\left.\mathrm{C}_{14}\right)$ increased from $\mathrm{I}$ 7.I to 24.9 per cent. The proportion of palmitic acid changes little. For the (B) cows the change was different ; in particular, the proportion of long-chain fatty acids increased (table r. fig. 1).

2. The free fatty acid content of plasma (NEFA) increased greatly at calving. It then changed in all cows in the same way as the proportion of long-chain fatty acids in milk fat changed. For the (A) cows it was from 3 to 5 times less in the sixth than in the first week of lactation. For the other cows it doubled between the first and the sixth week (table 2, fig. 2 and 4 ).

3. The changes in composition of milk fat seemed to be due to mobilization of lipid reserves, which was well demonstrated by the parallel changes in liveweight, energy balance and the longchain fatty acid content of milk seen in the same animal and also among animals (table 3 , fig. 3 and 4).

4. The fat content of the milk of most of the cows changed in the same way as the long-chain fatty acid content of milk but the relation of these two variables among individuals was weak (table 3 , fig. 3).

5. The free fatty acids in plasma may be important precursors of milk fat at the beginning of lactation. In a more general way their study in the milking cow seems to be particularly interesting especially to increase the understanding of the routes of lipid metabolism.

\section{RÉFÉRENCES BIBLIOGRAPHIQUES}

Balasse E., Rasio E., I965. Mécanisme d'action de l'ocytocine sur la concentration plasmatique des acides gras libres chez le chien. Arch. int. Pharmacodyn., 157, 356-359.

Barry J. M., Bartley W., Linzell J. L., Robinson P.S., ig63. The uptake from the blood of triglyceride fatty acids of chylomicra and low-density lipoproteins by the mammary gland of the goat. Biochem. J., 89, 6-I 1 .

DECAEN C., ADDA. J., Ig66. Évolution de la sécrétion des acides gras des glycérides du lait de vache au cours de la lactation. XVII Congr. internation. laiterie, A, 16 I-I $7 \mathrm{I}$.

Decaen C., Poutous M., Calomitis., I.967. Évolution de la production et de la composition du lait des vaches laitières au cours des deux premiers mois de lactation. Ann. Zootech. (sous presse)

DoLe V. P., 1956. A relation between non-esterified fatty acids in plasma and the metabolism of glucose. J. Clin. Invest., 35, I 50-1 54 .

Dole V. P., Meinertz Hanz, 1960. Micro determination of Iong-chain fatty acids in plasma and tissues. J. biol. Chem., 235, 2595-2599.

Dole V. P., RizaK Ma., I96r. On the turn over of long-chain fatty acids in plasma. J. Lipids Res., 2., 90-9I.

Duncan Wrh., Garton Ga., 1963. Plasma lipids of the cow during pregnancy and lactation. Biochem $J$., 89, 4I4-4I9.

Flate W. P., Moore L. A., Hooven N. W., Plowman R. D., I965. Energy metabolism studies with a high producing lactating dairy cow. Annu meeting am. Dairy Sci. Assoc., Lexington, Kentuky, Paper45.

Garton G. A., Duncan W. R., I964. Blood lipids, 5, The lipids of sheep plasma. Biochem. J.92, 472-479.

Glascock R. F., I958. Recent research on the origin of milk fat. Proc. r. Soc., Ser. B., 159, 402-420.

GoRdon R. S. (jr), Cherkes A. 1956. Unesterified fatty acid in human blood plasma. J. clin. Invest., 35, 206-2 $\mathrm{x} 2$.

Hartmann P. E., Lascelles A. K., I964. The uptake of plasma lipids and some non-lipid constituants by the mammary gland of the cow. Austra. J. biol. Sci., 17, 935-944.

Hartmanin P. E., Lascelles A. K., i965. Variation in the concentration of lipids and some other constituants in the blood of plasma of cows at various stage of lactation. Austral. J. biol. Sci., 18, II4-I23.

Hartmann P. E., Lascelles A.K., I.965. The effect of starvation on the uptake of the precursors of milk fat by the bovine mammary gland. Austral. J. biol. Sci., 18, 1025-1034.

Head H. H., Connoly J. P., Williams W. F., ig64. Glucose metabolisme in dairy cattle and the effect of acetate infusion. J. Dairy Sci., 12, 1271-1377.

Húth E. W., I960. Zur Frage des Physiologischen ben Rendern und Scheinen, Mariensee. 
Hutron J. B., ig63. The effect of lactation on intake in the dairy cow. Proc. New Zeal. Soc. anim. Prod., 23, $39-52$.

JoURNET M., JARRIGe R., 1960. Évolution de la sécrétion des matières grasses, des matières azotées et du lactose au cours du premier mois de lactation. Ann. Zootech., 9, I33-1 55 .

Knobil E., Hotchisiss J., 1964. Growth hormone. Amer. Rev. Physiol., 26, 47-74.

Kronferd D. S., I965. Plasma non-esterified fatty acid concentrations in the dairy cow : responses to nutritional and hormonal stimuli and significance in ketosis. Veter. Record., 77 , 30-35.

Lascelles A. K., Hardwick D. C., Linzell J. L., Mepham,., TB ig64. The transfer of ( $\left.{ }^{3} \mathrm{H}\right)$ stearic acid from chylomicra to milk fat in the goat. Biochem. J., 92, 36-42.

Lauryssens M., Verbecke R., Peeters G., I96I. Metabolisme of stearate I-C14 in the isolated cow's udder. J. Lipid Res., 2, 383-388.

McClymont G. L., Vallance S., 1962. Depression of blood glycerides and synthesis of milk fat by glucose infusion. Proc. Nutrit. Soc., 21, XLI.

Robinson D. S., r963. Change in the lipolytic activity of the guinea pig mammary gland at parturition. J. Lipid Res., 4, 21-23.

Salmon-LegagneUR E., 1965. Quelques aspects des relations nutritionnelles entre la gestation et la lactation chez la Truie. Thèse Doct. Paris.

Steinberg D., VaughaN M., I96r. Metabolic and hormonal régulation of mobilization of fatty acids from adipose tissue. Vth internation. Congr. Biochem., Moscov, p. 197.

Storry J. E., Rook J.A. F., I964. Plasma triglycerides and milk fat synthesis. Biochem. J., 91, 27 C-29 C. VAN SoEsT P. J., I963. Ruminant fat metabolism with particular reference to factors affecting low milk fat and feed efficiency. A review. J. Dairy Sci., 46, 204-216.

Williams W. F., WeisshaAR A. G., Lauterback G. E., 1965. Lactogenic hormone effects on plasma NEFA and blood glucose concentrations. Amer. Dairy Sci. Assoc. Section C p6o.

Winkler B., Steele R., Altszuler N., DE Bodo R. C., I964. Effect of growth hormone on free fatty acid metabolism. Amer. J. Physiol., 206, I 74-1 78. 